\title{
Exactly Integrable Analogue of a One-dimensional Gravitating System
}

\author{
Bruce N. Miller, Kenneth R. Yawn, and Bill Maier \\ Department of Physics and Astronomy, \\ Texas Christian University, \\ Fort Worth, Texas 76129
}

\begin{abstract}
Exchange symmetry in acceleration partitions the configuration space of an $N$ particle, onedimensional, gravitational system into $N$ ! equivalent cells. We take advantage of the resulting small angular extent of each cell to construct a related integrable version of the system that takes the form of a central force problem in $N-1$ dimensions. The properties of the latter, including the construction of trajectories, as well as several continuum limits, are developed. Dynamical simulation is employed to compare the two models. For a class of initial conditions, excellent agreement is observed.

PACS numbers: 05.10.-a, 05.45.-a , 56.25.EF , 05.70.Fh
\end{abstract}

Keywords: integrable dynamical system, $N$-body simulations, gravity, Vlasov equation 
One dimensional systems have played a central role in the development of physical models. Because of their relative simplicity, they form the starting point for the study of a wide variety of interactions. One-dimensional models of gases have been studied extensively in statistical mechanics and one-dimensional lattice models have been used in solid state physics to study metal alloys, magnetic spin systems, glasses, phonon propagation, electronic bands and a host of other systems. One dimensional gravitational systems have been used to investigate relaxation to equilibrium, phase transitions, the gravothermal catastrophe, and cosmology [1]. An important example is the system consisting of a one-dimensional chain of coupled, nonlinear, oscillators. Known as the Fermi-Pasta-Ulam (FPU) problem[2], it has had an enormous impact on the development of dynamics in the last fifty years. Its failure to come to thermal equilibrium in simulations on the earliest electronic computers stimulated intense research in the theory of conservative dynamical systems and resulted in major breakthroughs such as the KAM theorem and developments in soliton theory [2]. The analogous system in astronomy consists of $N$ parallel planar mass sheets of infinite extent that are restricted to move perpendicular to their surface. Like the FPU problem, this is a nonlinear, one-dimensional system. The only forces on a given sheet (or particle) are gravitational, and the system obeys the Poisson equation in one dimension. Since each particle experiences a constant acceleration until it encounters a neighboring particle, the motion can be expressed analytically and it is straightforward to simulate the system on the computer [3]].

The one dimensional self-gravitating system (OGS) was the first gravitational system to be investigated numerically 4] and, like the FPU system, it resists relaxing to statistical equilibrium. Early studies showed that the system virialized within 50 or so characteristic system crossing times, $\tau_{c}$, 4] as a consequence of "violent relaxation" 5]. Following this period the system appears to approximate a stationary Vlasov state which evolves extremely slowly, perhaps on the order of millions of $\tau_{c}$. Evidence of the approach to thermal equilibrium has only been recently established for a two component system [6, 7]. For an extensive review of the history of the relevant investigations see [1, 7]. In this paper we offer a possible explanation for the incredibly slow exploration of phase space these systems exhibit. We demonstrate analytically and with numerical simulation that an exactly integrable Hamiltonian system (EIS) exists which closely represents the OGS as its population increases. Further we derive a continuum (Vlasov) limit for the EIS and show that it is characterized 
by two integral invariants, as well as an exactly periodic radius. We also show that the continuum model can be extended to the pair space and provide information concerning correlations in position and velocity.

Consider an OGS consisting of $N$ parallel mass sheets (particles) of equal mass surface density $m$ and let the real $x$ axis be perpendicular to their surface. Label the sheets in terms of their increasing ordering from left to right so their positions are $x_{j}$ with $x_{j+1}>x_{j}(j=$ $1, \ldots, N-1)$. Then, from Gauss' law, the acceleration $A_{j}$ experienced by particle $j$ is a constant proportional to the net difference in the number of particles to its right and left:

$$
A_{j}=2 \pi m G(N+1-2 j) .
$$

As the system evolves, each particle undergoes uniform acceleration until a pair of particles crosses, at which time their accelerations are exchanged. Since the system is isolated from external forces, the total momentum and energy are conserved, while the center of mass moves with uniform velocity. Without loss of generality we may assume we are in a frame where the total momentum is zero and the center of mass is at rest. To establish a connection with the Vlasov limit, it is more convenient to represent the system in terms of positions and velocities $\left(x_{j}, v_{j}\right)$ of the particles. Thus

$$
\sum_{1}^{N} x_{j}=0, \sum_{1}^{N} v_{j}=0, E=\frac{m}{2} \sum_{1}^{N} v_{j}^{2}+2 \pi m^{2} G \sum_{i<j}\left|x_{i}-x_{j}\right|
$$

where $E$ is the total energy. It is also convenient to introduce a system of unit vectors, $\mathbf{e}_{j}$, in the $N$ dimensional configuration space. Then we can represent the system in terms of $N$ dimensional position, velocity (in the tangent space), and acceleration vectors,

$$
\mathbf{x}=\sum_{1}^{N} x_{j} \mathbf{e}_{j}, \mathbf{v}=\sum_{1}^{N} v_{j} \mathbf{e}_{j}, \mathbf{A}=\sum_{1}^{N} A_{j} \mathbf{e}_{j}
$$

In this context, the system is represented by a single point moving with constant acceleration until a crossing occurs, whereby two of the acceleration components are exchanged; i.e. the actual components of $\mathbf{A}$ depend on the ordering of the particle positions. Letting $\mathbf{e}_{c}=\frac{1}{N} \sum_{j} \mathbf{e}_{j}$, the constraints on the total momentum and the center of mass can be expressed by $\mathbf{v} \cdot \mathbf{e}_{c}=\mathbf{x} \cdot \mathbf{e}_{c}=\mathbf{0}$. As a result of the dynamical constraints (including energy conservation), the trajectories lie on a $2 N-3$ hyper-surface of the $2 N$ dimensional phase space. We 
can also introduce a generalized angular momentum tensor as a two form, or dyadic in the older formalism [8], and its square:

$$
\begin{gathered}
\mathbf{L}=\mathbf{x} \wedge m \mathbf{v}=m \sum_{i, j=1}^{N} x_{i} v_{j} \mathbf{e}_{i} \wedge \mathbf{e}_{j}=\frac{m}{\sqrt{2}} \sum_{i, j=1}^{N}\left(x_{i} v_{j}-x_{j} v_{i}\right) \mathbf{e}_{i} \mathbf{e}_{j} \\
L^{2}=\frac{m^{2}}{2} \sum_{i, j=1}^{N}\left(x_{i} v_{j}-x_{j} v_{i}\right)^{2} .
\end{gathered}
$$

It is straightforward to show that, for $N=3$, these definitions reduce to the familiar equations of three dimensional orbital mechanics. For completeness, and to compare with our further development below, we mention that the system has a well defined continuum (Vlasov) limit. By projecting the single point representing the system in its $2 N$ dimensional phase space onto a single $(x, v)$ plane, the system is represented by a cloud of $N$ points. If we let $N \rightarrow \infty$ while constraining the total mass, $M=N m$, and energy $E$, the system is described by a fluid in the $\mu(x, v)$ space with normalized distribution function $f(x, v ; t)$ satisfying the Vlasov (or Vlasov-Poisson) equation

$$
\begin{gathered}
\frac{\partial f}{\partial t}+v \frac{\partial f}{\partial x}+A[f] \frac{\partial f}{\partial v}=0 \\
A[f]=2 \pi M G \int d v^{\prime} \int d x^{\prime} f\left(x^{\prime}, v^{\prime}, t\right)\left[\Theta\left(x^{\prime}-x\right)-\Theta\left(x-x^{\prime}\right)\right]
\end{gathered}
$$

where $\Theta(x)$ is the usual step function [1, 7]. This system has been studied in the literature extensively and is known to have an infinite number of stationary solutions [1, 7, 9]. In particular, the maximum entropy solution is $f \sim \exp \left(-\beta v^{2} / 2\right) \cosh ^{-2}\left(2 \pi M^{2} G x / 2 E\right)$.

To understand our approach, we first consider a simpler system of a single particle moving in the plane in which we introduce the usual polar coordinates, $(r, \theta)$. We imagine that the plane is segmented into $P$ pie shaped "wedges" which share a common vertex at the origin and subtend $2 \pi / P$ radians. In the $k^{\text {th }}$ wedge the particle experiences a constant acceleration $\mathbf{g}_{k}$, with $\left|\mathbf{g}_{k}\right|=g,(k=1, \ldots, P)$, directed along the wedge bisector, thus pointing approximately towards the origin (common vertex, see Fig. 11). Then within each segment the particle executes a parabolic trajectory until it reaches a wedge boundary, which it crosses and begins a new parabolic segment with a rotated acceleration. If, as in Fig. 1, $P=6$ it has been shown that this system is isomorphic to the system of three parallel mass 
sheets [10]. Note that the constraint on the center of mass constrains the motion to the plane. We now imagine what happens as $P$ becomes large. In each wedge the acceleration is directed more nearly in the radial direction, i.e. towards the origin. Thus the system is very close to another one where the acceleration $\mathbf{g}=-g \mathbf{r} / r$ varies smoothly and is defined everywhere except at the origin. This latter system is exactly integrable: both energy and angular momentum are exactly conserved. For large, but finite, $P$, most orbits in the former system will lie close to their integrable counterparts for long times. However, for any $P<\infty$, there will be time intervals where $\frac{d \theta}{d t}$ changes sign for some of the orbits in the segmented system and the orbits will start to diverge from their integrable cousins.

Now let's shift our attention back to the OGS. Note that there are $N$ ! possible orderings of the particle labels. Associated with each ordering is a pyramidal segment in the configuration space in which the acceleration, $\mathbf{A}$, is a unique constant. To explore how the geometry changes with increasing $N$, let's compute $\cos (\phi):=\mathbf{A} \cdot \mathbf{A}^{*} / A^{2}$ where $\mathbf{A}^{*}$ has the permuted particle ordering to $\mathbf{A}$ obtained by interchanging the positions of a pair of adjacent particles, and $A^{2}=\sum A_{j}^{2}$. With a little work we obtain,

$$
\frac{2(2 \pi M G)^{2}}{N^{2} A^{2}}=\sin ^{2}(\phi / 2), A^{2}=\frac{1}{3}(2 \pi M G)^{2} N\left(1-N^{-2}\right) .
$$

Therefore, for $N \gg 1, \phi \cong 2 \sqrt{6} / N^{3 / 2}$ becomes vanishingly small, and we are faced with a similar situation to the planar example discussed above, i.e. there are $N$ ! cells within which the acceleration is approximately in the radial direction in the $N-1$ dimensional space obtained by projecting out the center of mass direction $\mathbf{e}_{c}$. As a consequence we can identify the OGS with a different system consisting of a single particle moving in $N$ dimensions with a radial acceleration of constant magnitude $A$. As we shall quickly see, the latter is an exactly integrable system. Let us signify the coordinates and velocities of the smooth system by $\left(y_{j}, u_{j}\right)$ so its position and velocity are $\mathbf{y}=\sum y_{j} \mathbf{e}_{j}, \mathbf{u}=\sum u_{j} \mathbf{e}_{j}$. Then the new equations of motion are

$$
\frac{d y_{j}}{d t}=u_{j}, \frac{d u_{j}}{d t}=-\frac{A y_{j}}{R}, R^{2}=\sum_{1}^{N} y_{j}^{2} .
$$

This is a central force problem in $N$ dimensions with a potential energy $\Phi=m A R$. Direct substitution in the above quickly shows that the energy, $H:=\frac{m}{2} u^{2}+\Phi$, the generalized angular momentum two form $\mathbf{L}=m \mathbf{y} \wedge \mathbf{v}$, and $L^{2}$ are exact dynamical invariants of the 


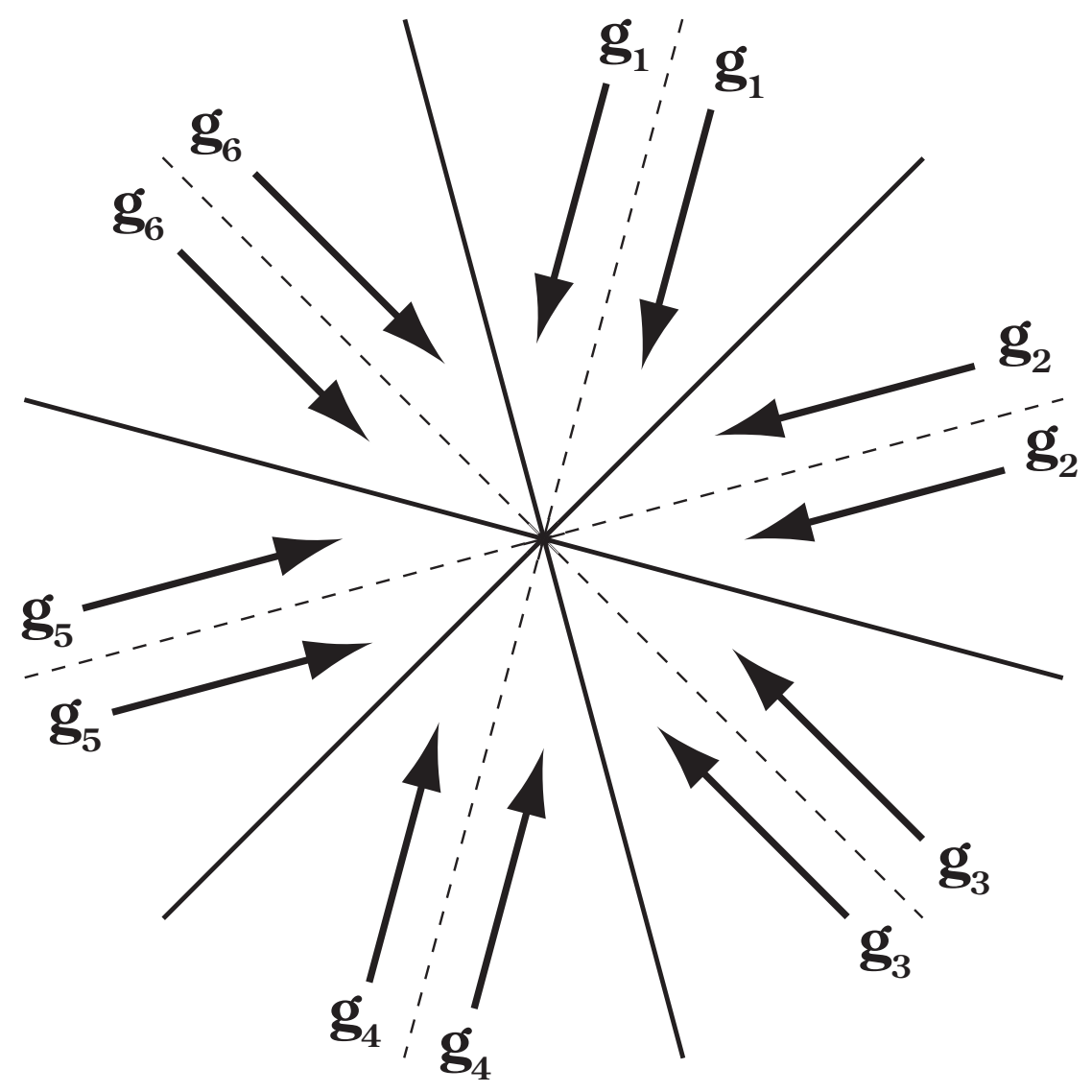

FIG. 1: Partition of the plane into six segments. Arrows indicate direction of acceleration. Note that within each wedge shaped segment the acceleration is pointing inward and exactly parallel to the wedge bisector.

motion. Moreover, if at an initial time, $\mathbf{e}_{c} \cdot \mathbf{y}=\mathbf{e}_{c} \cdot \mathbf{u}=0$, then by taking successive derivatives of $\mathbf{y}$ and $\mathbf{u}$ with respect to $t$ it can be seen that these quantities don't change their value, and the trajectories remain constrained to an $N-1$ dimensional manifold. It is remarkable that, in spite of the arbitrary number of dimensions, since $\frac{d \mathbf{u}}{d t} \sim \mathbf{y}$, the motion remains in the two-plane defined by the initial vectors $\mathbf{y}_{0}$ and $\mathbf{u}_{0}$ ! Therefore the motion is simple to 
describe: If we impose polar coordinates $(R, \theta)$ in the plane of the motion we obtain

$$
\frac{d^{2} R}{d t^{2}}=-A+\frac{L^{2}}{m^{2} R^{3}} \quad, \quad \frac{d \theta}{d t}=\frac{L}{m R^{2}}
$$

Alternatively, a first order equation for $R(t)$,

$$
\frac{d R}{d t}= \pm \sqrt{\frac{2}{m}\left[H-m A R-\frac{L^{2}}{2 m R^{2}}\right]},
$$

can be obtained directly from the energy equation and shows that turning points of the orbit occur where the argument of the radical vanishes. Consequently $R(t)$ is a periodic function of the time with period, say, $\tau_{R}$. However, we must be careful to note that $\theta(t)$ does not advance by $2 \pi$ radians in the time $\tau_{R}$, so the orbits are not closed in space. By inverting the equation (solving for $t=t(R)$ ) it can be shown that the solution is a linear combination of elliptic integrals.

To obtain the complete set of coordinates and velocities, we use the initial position and velocity, $\mathbf{y}_{0}=\mathbf{y}(t=0), \mathbf{u}_{0}=\mathbf{u}(t=0)$, to define orthogonal unit vectors in the plane, $\mathbf{b}_{1}$, $\mathbf{b}_{2}$ :

$$
\mathbf{b}_{1}=\frac{\mathbf{y}_{0}}{R_{0}}, \mathbf{b}_{2}=\frac{\left(\mathbf{u}_{0}-\left(\mathbf{u}_{0} \cdot \mathbf{e}_{y}\right) \mathbf{e}_{y}\right)}{\left(u_{0}^{2}-\left(\mathbf{u}_{0} \cdot \mathbf{e}_{y}\right)^{2}\right)} .
$$

Then we can impose Cartesian coordinates $\left(z_{1}, z_{2}\right)$ such that

$$
\mathbf{y}(t)=z_{1} \mathbf{b}_{1}+z_{2} \mathbf{b}_{2}, \mathbf{u}(t)=\frac{d z_{1}}{d t} \mathbf{b}_{1}+\frac{d z_{2}}{d t} \mathbf{b}_{2}
$$

where $\left(z_{1}, z_{2}\right)$ satisfy

$$
\frac{d^{2} z_{1}}{d t^{2}}=-A \frac{z_{1}}{\sqrt{z_{1}^{2}+z_{2}^{2}}}, \frac{d^{2} z_{2}}{d t^{2}}=-A \frac{z_{2}}{\sqrt{z_{1}^{2}+z_{2}^{2}}} .
$$

The complete set of coordinates and velocities are obtained from $y_{j}(t)=\mathbf{e}_{j} \cdot \mathbf{y}(t)$, $u_{j}(t)=\mathbf{e}_{j} \cdot \mathbf{u}(t)$. Note that the Cartesian pair $\left(z_{1}, z_{2}\right)$ can be obtained either by solving this pair of coupled second order equations, or by simply noting that $z_{1}=R \cos \theta, z_{2}=R \sin \theta$.

Several continuum limits for the exactly integrable gravitational analogue (EIS) can be constructed by focusing on $R^{2}=\sum y_{j}^{2}$. As for the OGS, project the phase space onto a single two dimensional plane $(y, u)$ to obtain a cloud of $N$ points, and take the limit $N \longrightarrow \infty$ while constraining the total mass, $M=N m$, and energy $H$. Let $f_{I}(y, u ; t)$ be the normalized 
density pdf in the $(y, u)$ plane. Then in the continuum limit we can immediately identify $R^{2}=\sum y_{j}^{2}$ with $N \int d y \int d u y^{2} f_{I}:=N\left\langle y^{2}\right\rangle$ which, in general, depends on the time. We will see that in the large $N$ limit the factors of $N$ will scale out of the equations. Recalling that $A \sim \sqrt{N}$, the equations of motion of a point in the $(y, u)$ plane in the continuum limit are

$$
\frac{d y}{d t}=u, \frac{d u}{d t}=-\frac{A y}{\sqrt{N\left\langle y^{2}\right\rangle}}=-\frac{(2 \pi M G) y}{\sqrt{3\left\langle y^{2}\right\rangle}},
$$

which results in the following Vlasov equation for $f_{I}(y, u, t)$ :

$$
\frac{\partial f_{I}}{\partial t}+u \frac{\partial f_{I}}{\partial y}-\frac{(2 \pi M G) y}{\sqrt{3\left\langle y^{2}\right\rangle}} \frac{\partial f_{I}}{\partial u}=0 .
$$

It's important to keep in mind that, because of the dependence of $\left\langle y^{2}\right\rangle$ on $f_{I}(y, u$; $t$ ) (it is a functional), this is not simply the equation of a distribution of independent harmonic oscillators.

We have seen that explicit $N$ dependence disappears from the equations of motion in the fluid limit. In the continuum limit it's convenient, and in fact necessary, to introduce scaled quantities $r, h, a, l^{2}$,

$$
r:=\frac{R}{\sqrt{N}}, h:=\frac{H}{m N}, a:=\frac{A}{\sqrt{N}}, l^{2}:=\frac{L^{2}}{m^{2} N^{2}} .
$$

Then their limiting values are

$$
r^{2}=\left\langle y^{2}\right\rangle, h=\frac{1}{2}\left\langle u^{2}\right\rangle+a r, a=\frac{2 \pi M G}{\sqrt{3}}, l^{2}=\left\langle y^{2}\right\rangle\left\langle u^{2}\right\rangle-\langle y u\rangle^{2} .
$$

A remarkable feature of the fluid limit is that the radial equation is still preserved, i.e. substituting the scaled variables gives the identical differential equation in terms of the scaled variables, so that $r(t)$ is still a periodic function of time. With a little work, we find from the Vlasov equation for $f_{I}$ that both $h$ and $l^{2}$ are integral invariants of the fluid motion defined by Eq. (15). In common with the OGS, there are an infinite number of stationary solutions of Eq.(16). We can construct a family of such solutions by defining the combined energy-like function (and functional) $g$ :

$$
g\left(y, u,\left[f_{I}\right]\right):=\frac{1}{2} u^{2}+\frac{1}{2} \frac{a y^{2}}{\sqrt{\left\langle y^{2}\right\rangle}} .
$$


Note that $\frac{\partial g}{\partial u}$ is the local velocity and $-\frac{\partial g}{\partial y}$ is the local acceleration. Therefore any integrable functional of $g$ satisfies the Vlasov equation and yields a stationary solution $f_{I}[g]$. Note also that $\langle g\rangle \neq\langle h\rangle$. It is also possible to use the invariance of $h$ and $l^{2}$ to construct the family of maximum entropy solutions.

The standard derivation of the Vlasov equation starts with the BBGKY hierarchy for a system of pairwise interacting particles and proves that, in the Vlasov limit, the higher order distribution functions factor into products of the singlet distribution $f$ [1] , so that pairs of particles become statistically independent. In fact, we implicitly made this assumption in determining the expression for $l^{2}$. However, in contrast with the OGS, the EIS does not have pairwise additive interactions so it may be possible to construct additional continuum representations which include correlations. This possibility is suggested by the form of the generalized angular momentum, Eq.(44), which depends on the coordinates and velocities of pairs of particles. Note that, in the continuum limit, both $l_{p}:=\left(y u^{\prime}-y^{\prime} u\right)$ and its square are invariants of the motion. Let $f_{I}^{(2)}\left(x, u ; x^{\prime}, u^{\prime} ; t\right)$ be a normalized distribution in the four dimensional pair space and assume that it satisfies the extended Vlasov equation

$$
\frac{\partial f_{I}^{(2)}}{\partial t}+u \frac{\partial f_{I}^{(2)}}{\partial y}+u^{\prime} \frac{\partial f_{I}^{(2)}}{\partial y^{\prime}}-\frac{(2 \pi M G) y}{\sqrt{3\left\langle y^{2}\right\rangle}} \frac{\partial f_{I}^{(2)}}{\partial u}-\frac{(2 \pi M G) y^{\prime}}{\sqrt{3\left\langle y^{\prime 2}\right\rangle}} \frac{\partial f_{I}^{(2)}}{\partial u^{\prime}}=0
$$

Then, as in the standard formulation, one class of solutions can always be constructed by the product $f_{I}(y, u ; t) f_{I}\left(y^{\prime}, u^{\prime} ; t\right)$. However, there are now other possibilities. For example, the integrable functionals of $l_{p}$ (e.g. $f_{I}^{(2)} \sim \exp \left(-c l_{p}^{2}\right)$ ) will generate a family of stationary solutions in which pairs of points are obviously correlated. It will be interesting to look for connections with earlier studies of correlation in the OGS [12].

Of course, due to their dynamical instability, nearly all orbits generated by the OGS will diverge from their EIS counterparts sooner or later. To get an idea of how well the orbits generated by the EIS follow the those of the OGS, we carried out numerical simulations of the OGS and integrated the radial equation numerically for the EIS. For the initial conditions we selected random initial positions and velocities that are uniformly distributed in a rectangle in $(x, v)$. We chose units of mass and time where $M:=m N=1$ and $2 \pi G=1$.We made small translations to guarantee that the total momentum and center of mass are null, and scaled the positions and velocities so that the total energy is exactly 0.75 and the virial ratio (twice the kinetic energy divided by the potential energy) is exactly 1.0. In each case we 


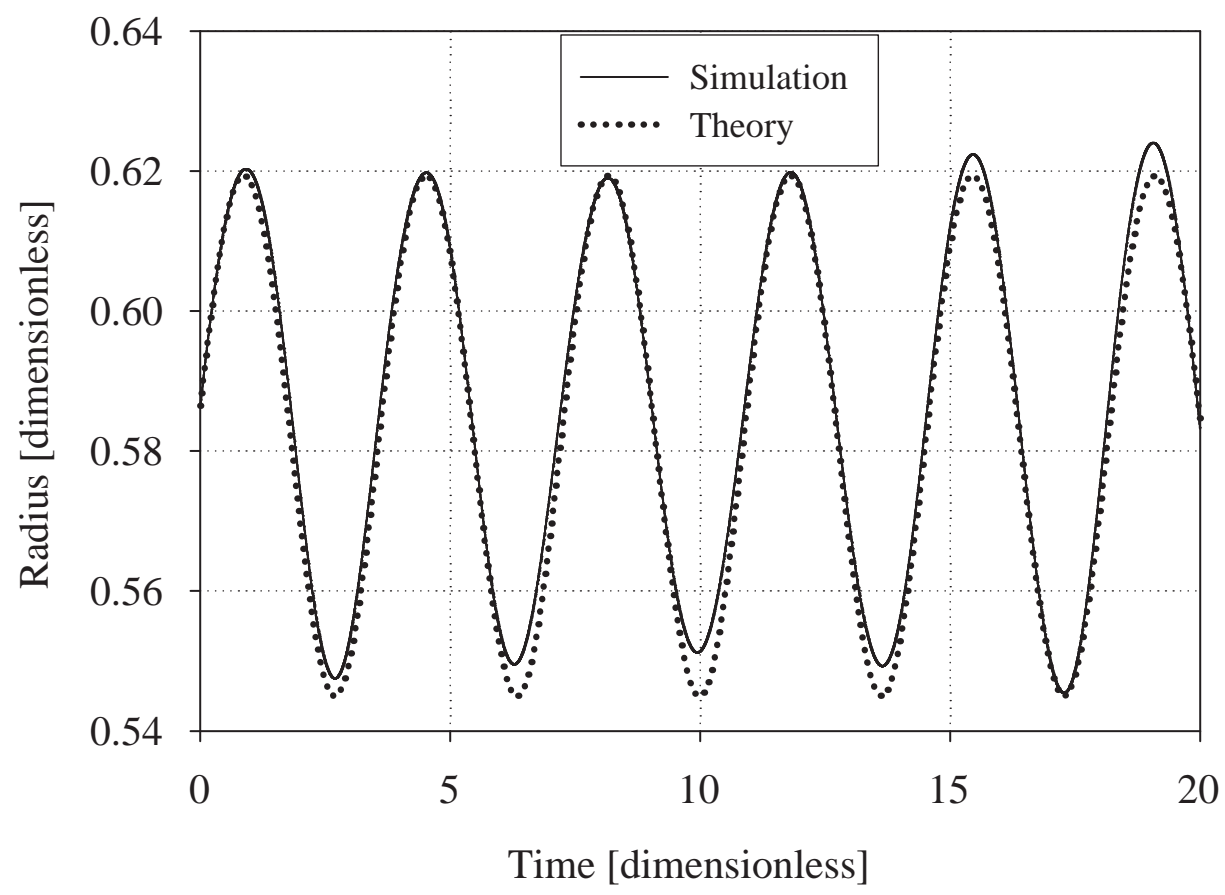

FIG. 2: Comparison of the radial coordinate $R(t)$ computed for the OGS and EIS with the same initial condition. Note the close agreement for this initial condition.

computed the initial values of $R^{2}$ and $L^{2}$ which are all that is required to solve the radial equation, Eq.(11).

Below we present graphs comparing the theory, from the EIS, and simulations. Although the results are not exhaustive, there seems to be a well established trend. In cases where the simulations exhibit a very regular variation of the radius, there is a remarkable agreement between theory (as given by the EIS) and simulation. This can be seen in Figure 2 . For other, equally likely, initial conditions the simulated $R(t)$ is not regular and, therefore, is not well represented by the theory (see Figure 3 ). However, even in these cases, we find that the maxima and minima of the simulations line up fairly closely with those of the theory, i.e. they are in phase. This seems to be a general trend whether we are modelling the initial behavior or letting the system anneal for a while before comparisons are made.

Starting from the $N$ particle one-dimensional self-gravitating system (OGS), we have shown how to construct an exactly integrable Hamiltonian system of arbitrary dimension (EIS) in which the motion is always confined to a plane in the configuration space. The system consists of a single point which experiences an acceleration of constant magnitude 


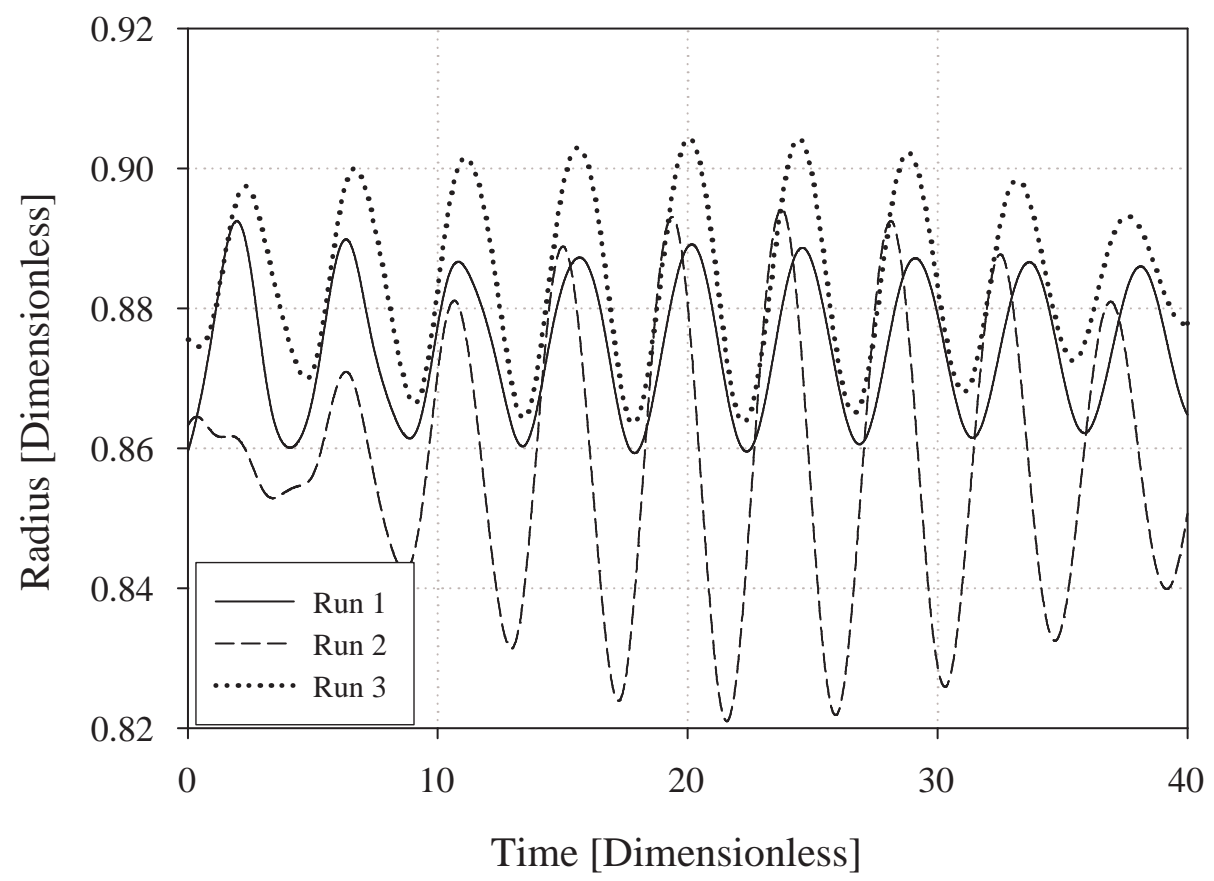

FIG. 3: Evolution of the radial coordinate for the OGS from three different initial conditions selected using the identical algorithm.

that is always directed towards a fixed point (say the origin) of $N$ dimensional Euclidean space. The exact solution of the equations of motion can be expressed as a linear combination of elliptic integrals. In general, the motion fills an annulus in the plane between two turning points, but degenerate cases are possible where the motion is exactly circular (maximum possible angular momentum), resulting in stationary motion, or linear (null angular momentum), resulting in the periodic collapse and expansion of the system. By imposing the Vlasov limit, we have shown how to construct two types of continuum models for the EIS, one of which is capable of representing correlations between particles. Simulations of the OGS have been compared with solutions of the EIS with identical initial conditions. For cases where the dynamical simulations are regular, the agreement between the two models is outstanding. For other less regular behavior, extrema in the simulations seem to occur at similar times as the periodic extrema of the integrable model, so the solutions are in phase. From the preliminary results obtained so far, it appears that the existence of substructure in the gravitational system is responsible for the lack of regularity. Further work concerning the influence of population, the graininess of distributions, and the theory and possible 
applications of the continuum representations of the EIS is ongoing. In addition we are investigating the extension of the theory to higher dimensional systems.

\section{Acknowledgments}

The authors benefitted from conversations with John Hopkins, George Gilbert, Tony Burgess, and Igor Prokhorenkov, as well as the support of the Research Foundation and Department of Information Services of Texas Christian University.

[1] B. N. Miller, Transport Theory and Statistical Physics, (2005), in Press.

[2] T. P. Weissert, The Genesis of Simulation in Dynamics (Springer-Verlag, New York, 1997).

[3] A. Noullez, D. Fanelli, and E. Aurell, cond-mat/0101336v1 (2001).

[4] L. Cohen and M. Lecar, Bull. Astr. 3, 213 (1968).

[5] D. Lynden-Bell, Mon. Not. R. Astron. Soc. 136, 101 (1967).

[6] K. Yawn and B. Miller, Phys. Rev. Letters 79, 3561 (1997).

[7] K. R. Yawn and B. N. Miller, Physical Review E 68, 056120 (2003).

[8] T. Frankel, The Geometry of Physics (Cambridge University Press, Cambridge, UK, 1997).

[9] S. Cuperman, A. Harman, and M. Lecar, Astrophys. Sp. Sci. 13, 397 (1971).

[10] H. E. Lehtihet and B. N. Miller, Physica D 21, 93 (1986).

[11] W. Braun and K. Hepp, Commun. Math. Phys. 56, 101 (1977).

[12] Monaghan, Aust. J. Phys. 31, 95 (1978). 